Published in J. Low Temp. Phys. 93, 1059-1067 (1993).

\title{
Creation of Quantized Vortices at the Lambda Transition in Liquid Helium-4
}

\author{
P C Hendry, N S Lawson, R A M Lee, P V E McClintock \\ and C D H Williams*
}

School of Physics and Materials, Lancaster University, Lancaster, LA1 4YB, UK.

* Department of Physics, University of Exeter, Exeter, EX4 4QL, UK.

A fast $(\sim 3 \mathrm{~ms})$ adiabatic expansion of a volume of liquid ${ }^{4}$ He through the lambda transition is being used to study the nature of the transition and to model the cosmological false vacuum to true vacuum phase transition of the early universe. Preliminary results are reported showing that, in accordance with theoretical predictions by W H Zurek (Nature 317, 505; 1985), there is copious production of quantized vortex lines, which represent the superfluid analogue of cosmic strings. The line density after the expansion appears to decay in two distinct stages, with a fast decay being followed by a much slower one, in agreement with earlier work on the decay of quantum turbulence created in thermal counterflow. Extrapolation of the initial fast decay suggests an initial line density, immediately following the expansion, of $\sim 10^{7} \mathrm{~cm}^{-2}$. Smaller, but still substantial, vortex densities are also found to occur when the system is expanded from below the lambda transition, and the physical implications are discussed.

\section{INTRODUCTION}

The origin of the vortex lines ${ }^{1}$ that seem to be universally present ${ }^{2}$ in He II represents an interesting question to which the answer remains incomplete. These vortices are extremely important because they are believed to seed the creation of the array of parallel vortex lines that appear when a vessel of He II is rotated, as well as the dense random tangles of vortex line that can be generated in a thermal counterflow. ${ }^{3}$ Awschalom and Schwarz found ${ }^{2}$ that remanent vorticity seemed to appear at the lambda transition and to decay towards a constant, presumed metastable, density that was apparently independent of the history of the cooling process.

One possible way ${ }^{2}$ in which vortices might be created at the lambda transition relates to the flow and convection that may be occurring in the liquid above the transition. Any such motions will effectively be converted to quantized vortex lines with the onset of long-range order, as the system cools through the transition. A second, quite different, mechanism relates to critical fluctuations of the superfluid 
order parameter close to the transition, which must also correspond to vortices. ${ }^{4}$ The latter process is of some topical interest in view of the suggestion ${ }^{5}$ that it may provide a basis for demonstrating, in the laboratory, the Kibble mechanism ${ }^{6}$ for creation of the topological defects that seeded galaxy formation.

The analogy between liquid helium and the early universe arises as follows. Viewed in terms of Ginzburg-Landau theory, ${ }^{7}$ the lambda transition may be considered as though it were a phase transition of second order, with a potential contribution to the free energy of form

$$
V=\alpha|\psi|^{2}+\frac{1}{2} \beta|\psi|^{4}
$$

where the complex order parameter $\psi$ is given by a solution of the GinzburgPitaevskii equation. Above the transition, the equilibrium value of $|\psi|=0$ (although there will also be thermal fluctuations to finite values: see below).Below the transition, however, $|\psi|$ acquires a finite equilibrium value and $V[\operatorname{Re}(\psi), \operatorname{Im}(\psi)]$ takes on the same "sombrero hat" shape as the corresponding cosmological free energy expressed in terms of Higgs fields. In the early universe, a symmetry-breaking phase transition from false vacuum to true vacuum is believed to have occurred $\sim 10^{-34}$ s after the big bang, once the temperature had fallen $\sim 10^{27} \mathrm{~K}$. Although there are many variants of the basic model, both with and without inflation, it is believed that a variety of topological defects ${ }^{8}$ would have been produced in the transition because of an event horizon that prevented adjacent regions from being causally connected. One type of defect, which appears to have the appropriate properties for it to have played a role in galaxy formation, is the cosmic string - a thin tube of false vacuum. The analogous object in He II is a quantized vortex line.

The idea of using the lambda transition to model the cosmological phase transition was due to Zurek. His suggestion ${ }^{5}$ was that a volume of He I should be expanded fast from an initial temperature and pressure $\left(T_{i}, P_{I}\right)$ to final values $\left(T_{f}, P_{f}\right)$, as sketched in Fig 1, so that it would undergo the transition to He II while still retaining frozen-in fluctuations from above the lambda point. The nascent superfluid would therefore form with a spatially incoherent order parameter, corresponding to a large density of vortex lines, modelling the cosmic strings of the early universe. This scenario depends on the fact that the liquid can (in principle) be expanded at any velocity up to that of first sound, whereas the propagation velocity for changes in the order parameter is equal to the (much slower) velocity of second sound.

In this paper, we report the initial results obtained from an expansion cryostat which we have built to carry out Zurek's proposed experiment ${ }^{5}$ and to test his predictions.

\section{EXPERIMENTAL DETAILS}

A full description of the expansion cryostat will be given in a later paper but, briefly, the arrangement was as follows. A sample of $\sim 10^{-3} \mathrm{~kg}$ of isotopically pure ${ }^{9}$ liquid ${ }^{4} \mathrm{He}$ is held within a small chamber whose walls consist of a bronze bellows, as indicated diagrammatically in Fig 2. The chamber is secured at the top, but its bottom surface can be pulled up to compress the liquid, or released to expand it as shown by the arrows, using a mechanical linkage from the top of the cryostat. 
The chamber is held within an evacuated enclosure immersed in a cryogenic bath of liquid ${ }^{4} \mathrm{He}$ at $\sim 2.0 \mathrm{~K}$. The cell is fitted with a capacitance pressure gauge and a carbon resistance thermometer. The temperature of the sample can be adjusted by means of a breakable thermal link to the surrounding bath, or by a heater.

To detect the vortices that are expected ${ }^{5}$ to be produced in an expansion, there is a small insert in the cell carrying a heater and bolometer for the generation and detection of second sound. The bolometer signal passes via a FET preamplifier in the bath at $\sim 2.0 \mathrm{~K}$, and thence to a main amplifier at room temperature. At the moment of the expansion, a sequence of second sound pulses is initiated, and their attenuation in the $\sim 4 \mathrm{~mm}$ of He II separating bolometer and heater provides a measure ${ }^{1}$ of the vortex line density. Typically, the cell is expanded from an initial pressure $P_{i}=29.6$ bar and temperature $T_{i}=1.81 \mathrm{~K}$, through the lambda transition, to final values of $P_{f}=6.9$ bar and $T_{f}=2.04 \mathrm{~K}$, in a time $\tau_{e} \sim 3 \mathrm{~ms}$. During the following $1.7 \mathrm{~s}$, some 170 single-pulse second sound signals are recorded by a Nicolet 1280 data processor. About $600 \mathrm{~s}$ later, at exactly the same pressure and temperature, a reference signal is averaged and recorded, defining the signal amplitude in the (virtual) absence of vortices.

The signal sequence is analysed automatically in two quite separate ways. First, any given signal is cross-correlated with the reference signal; the ratio of the maximum of the cross-correlogram to that of the auto-correlogram of the reference signal gives the ratio of the signal magnitude to that of the reference signal. Secondly, a least squares method is used to find the factor by which the reference signal must be multiplied in order to obtain a best fit to the signal being analysed, after each has first been baseline-corrected to an average of zero.

\section{RESULTS}

Some typical second sound signals, recorded shortly after an expansion, are shown in the inset to Fig 3. Although the signals at the shortest times (lower signal in each inset) are rather noisy, owing to mechanical vibrations caused by the expansion itself, coupled to a slight microphony of the low level electronics, it is immediately evident that the expansion leads to a considerable increase in attenuation. This is true both of expansions that take the system through the lambda transition, and of ones that start from below the transition i.e. with the sample already superfluid; but the attenuation is larger in the former case.

The evolution of the signal magnitude, expressed as a percentage of the reference signal, is shown as a function of time in the main part of Fig 3. The circled data points are for an expansion through the transition, and the triangles show what happens in an expansion from almost the same starting conditions but just below the transition. The results shown were all based on the least squares signal analysis; those from the correlation analysis were in agreement within experimental error and seldom differred by more than $2 \%$.

To enhance the signal/noise ratio, groups of adjacent signals were averaged together prior to analysis. This reduced, but did not eliminate, the effect of noise due to the mechanical vibrations; inevitably, the process was least effective where it was most needed, at very short times, where the size of the averaging groups had to be kept small (or equal to unity) to prevent distortion. The results shown in 
Fig 3 have all been enhanced in this way, reducing the scatter of the data but not causing any detectable systematic shifts.

\section{DISCUSSION}

It must be emphasized that the results presented in the preceding section are of a preliminary character. Data of higher quality are to be anticipated once the microphony of the cryogenic preamplifier system has been reduced and other improvements incorporated, including better temperature control and thermal isolation of the sample. Nonetheless, it is of interest to discuss the initial results in the light of the theoretical predictions and to draw some tentative conclusions.

The large transient second sound attenuation observed after the system has followed an expansion trajectory through the lambda transition (circled data in Fig 3 ) is qualitatively in excellent accord with Zurek's prediction. ${ }^{5}$ It must be borne in mind, however, that the geometry of the expansion is far from being ideal. In principle, to entirely avoid vortex creation by fluid flow, one would wish to contrive a spherically symmetrical expansion, or a longitudinal expansion of a cylinder with stretchable walls, but this appears to be impossible in practice. The actual cell approximates the latter situation, but both the convolutions of the bellows and also the presence of the second sound heater and bolometer within the cell represent nonidealities that will inevitably result in some fluid movement parallel to surfaces during the expansion. The expansion results in the bottom of the cell dropping a distance of $\sim 4 \mathrm{~mm}$ in $\sim 3 \mathrm{~ms}^{-1}$. Although the resultant transient velocity is quite fast (average $\sim 1 \mathrm{~ms}^{-1}$ ), we may note that there is no relative motion at all between the top and bottom surfaces of the cell and helium adjacent to them; the flow that occurs will presumably be due to the second order effects mentioned above. It is not obvious how to calculate the vortex line density likely to be produced by such flows, especially in view of their very short duration, but we may guess that it is likely to be considerably smaller than that expected to be produced through the Zurek mechanism. ${ }^{5}$

To try to estimate the vortex line density immediately following the expansion by extrapolation of the data back to time $t=0$, we note that the spontaneous decay rate $d L / d t$ of homogeneous superfluid turbulence is proportional ${ }^{10}$ to $-L^{2}$, where $L$ is the length of vortex line per unit volume. Using the same nomenclature and symbols as Schwarz and Rozen ${ }^{11}$ and the earlier papers by Schwarz, ${ }^{12}$ we may write

$$
\frac{d L}{d t}=-\alpha I_{l} \beta c_{L}^{-1} L^{2}
$$

where the constants $\alpha, I_{l}, \beta$ and $c_{L}^{-1}$ are all known, at least within limits, from earlier work on turbulence generated in thermal counterflow, and its free decay. Integrating (2), we find immediately that the evolving line length per unit volume is given by

$$
L^{-1}=\alpha I_{l} \beta c_{L}^{-1} t+L_{i}^{-1}
$$

where $L_{i}$ is the initial line density at $t=0$, which we take to be the instant when the expansion stops. The relationship between the line density and the attenuation 
of a second sound signal is known ${ }^{1}$ from experiments on rotating helium, and for present purposes can be expressed in the form

$$
L=\frac{8 u_{2} \ln \left(S_{0} / S\right)}{3 B \kappa d}
$$

where $u_{2}$ is the velocity of second sound, $S_{0}$ and $S$ are the signal amplitudes in the absence and presence of vortices, $B$ is a temperature dependent parameter ${ }^{1}, \kappa=$ $9.97 \times 10^{-4} \mathrm{~cm}^{2} \mathrm{~s}^{-1}$ is the quantum of circulation, and $d$ is the heater/bolometer separation. From (3) and (4) it is evident that a plot of $\left[\ln \left(S_{0} / S\right)\right]^{-1}$ as a function of $t$ should yield a straight line from whose ordinate intercept $L_{i}$ can be determined. Such a plot is shown in Fig 4 for the initial, rapidly rising, part of the evolution. From the intercept of a line fitted to the data by the method of least squares, we calculate that $L_{i} \sim 10^{7} \mathrm{~cm}^{-2}$. This is a very large value: to place it in context, we may note that to create a comparable density by rotation of a container of He II would require an angular velocity of $\sim 4000$ radians s $^{-1}$. It seems implausible that a line density of this magnitude could be created in the very brief episode of fluid flow during the expansion, and we conclude that it must arise mainly through the Zurek mechanism. ${ }^{5}$

It is evident from Fig 3 that smaller, but still very large, initial line densities are created by expansions that do not pass through the lambda transition (from the triangle data we obtain $L_{i}=2 \times 10^{6} \mathrm{~cm}^{-2}$ ). Again, there will obviously be a contribution from vortices produced by flow, but the density seems implausibly large to account for entirely in this way. A possible explanation can be found in Williams theory ${ }^{4}$ of the lambda transition as a vortex-driven phenomenon. At the starting point, very close to the lambda transition $T_{\lambda}$, there will be a relatively large equilibrium density of vortices. Following the expansion, the line density will therefore decay towards a new, lower, equilibrium value appropriate to the larger final value of $\left(T_{\lambda}-T\right)$. Thus, the experiment can perhaps be regarded as a rather direct way of revealing the large vortex density predicted to exist close to $T_{\lambda}$.

The growth of the signal following both types of expansion (Fig 3) exhibits evidence of a small local maximum or anomaly near $t=200 \mathrm{~ms}$, separating a regime of fast vortex decay from a second regime characterised by a much slower decay rate. Similar effects have been seen previously in the decay of turbulence generated by thermal counterflow, ${ }^{11,13,14}$ though not ${ }^{14}$ in the turbulence generated mechanically. The effect has been discussed ${ }^{11}$ by Schwarz and Rozen.

\section{CONCLUSION}

The preliminary results of the expansion experiment, reported above, are consistent with Zurek's prediction ${ }^{5}$ that a fast adiabatic passage through the lambda transition would result in copious vortex production. In common with some recent experiments on phase transitions in liquid crystals, ${ }^{15,16}$ these observations provide support for the ideas underlying the Kibble mechanism of galaxy formation. ${ }^{6,8} \mathrm{Be}-$ cause the velocity of second sound tends to zero at $T_{\lambda}$, it is to be expected that slower expansions, or temperature sweeps, through the transition will also lead to significant vortex creation which, in the latter case, would contribute to the remanent vorticity observed earlier. ${ }^{2}$ The smaller, but still large, vortex densities created 
by expansions from just below $T_{\lambda}$ may represent confirmation of the existence of a large equilibrium vortex density ${ }^{4}$ corresponding to critical fluctuations of the order parameter close to the transition.

Further investigations are now in progress to explore the influence of the expansion rate, the fractional molar volume change in the expansion, the starting temperature for expansions from below $T_{\lambda}$, and other relevant parameters.

\section{ACKNOWLEDGEMENTS}

Stimulating correspondence with W H Zurek is gratefully acknowledged. The research was supported by the Science and Engineering Research Council (UK).

\section{REFERENCES}

1. R.J. Donnelly, Quantized Vortices in Helium II, Cambridge University Press, 1991.

2. D.D. Awschalom and K.W. Schwarz, Phys. Rev. Lett. 52, 49 (1984).

3. J.T. Tough, in Progress in Low Temperature Physics, ed. D.F. Brewer, vol VIII, NorthHolland, Amsterdam, 1982, pp 133-219.

4. G.A. Williams, J. Low Temp. Phys. 89, 91 (1992).

5. W.H. Zurek, Nature 317, 505 (1985); and Acta Polonica to be published.

6. T.W.B. Kibble, J. Phys. A 9, 1387 (1976).

7. J. Tilley, Superfluidity and Superconductivity, Hilger, Bristol, 1986.

8. A. Vilenkin, Phys. Rep. 121, 263 (1985).

9. P.C. Hendry and P.V.E. McClintock, Cryogenics 27, 131 (1987)

10. H.E. Hall and W.F. Vinen, Proc. Roy. Soc. A238, 204 (1956).

11. K.W. Schwarz and J.R. Rozen, Phys. Rev. Lett. 66, 1898 (1991).

12. K.W. Schwarz, Phys. Rev. B 31, 5782 (1985).

13. W.F. Vinen, Proc. Roy. Soc. 242, 493 (1957).

14. M.R. Smith, R.J. Donnelly, N. Goldenfeld and W.F. Vinen, "Decay of vorticity in homogeneous turbulence", to be published.

15. I. Chuang, N. Turok and B. Yurke, Phys. Rev. Lett. 66, 2472 (1991).

16. M.Y. Bowick, L. Chander, E.A. Schiff and A.M. Srivastava, "The cosmological Kibble mechanism in the laboratory: string formation in liquid crystals", to be published. 


\section{Figures}

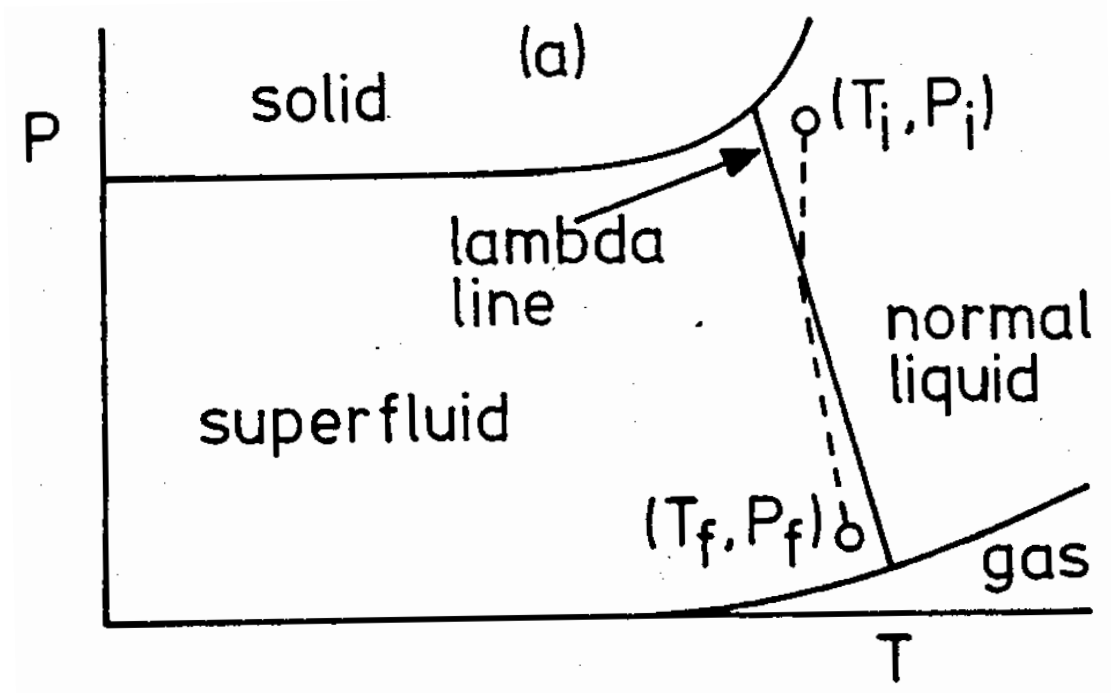

Figure 1: Sketch of expansion trajectory (dashed) through the lambda transition on the ${ }^{4}$ He phase diagram.

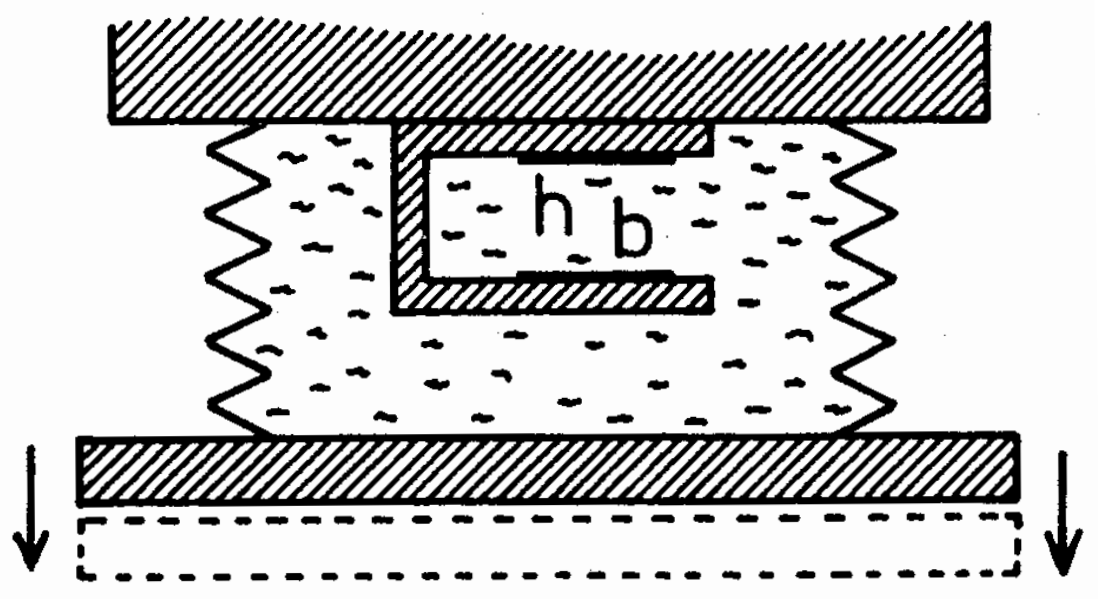

Figure 2: The experimental cell (schematic). 


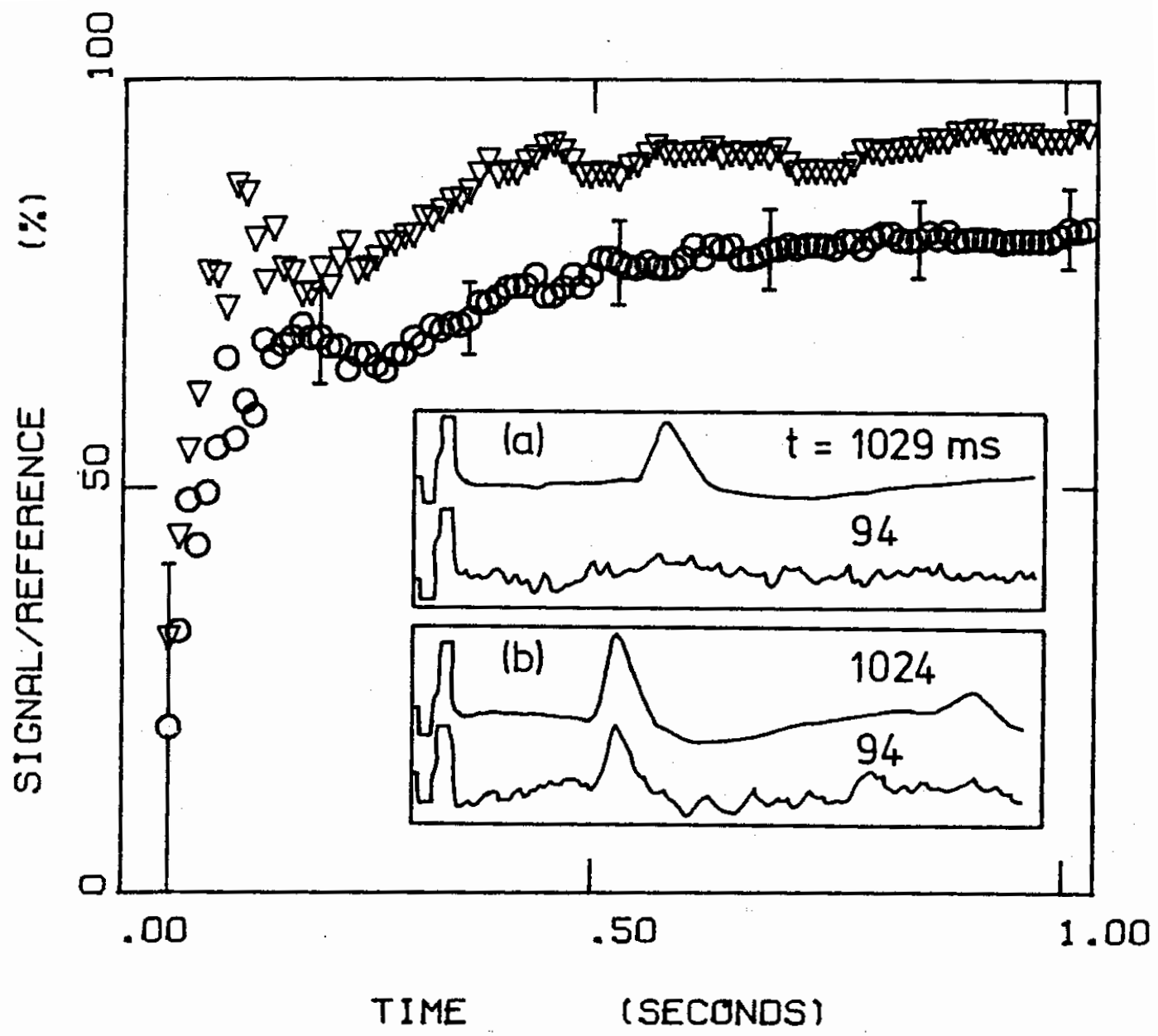

Figure 3: Evolution of the amplitude of second sound signals following the expansion at $t$ $=0$. The insets show typical signals measured at two different times for expansions: (a) through the transition; and (b) starting below the transition. 


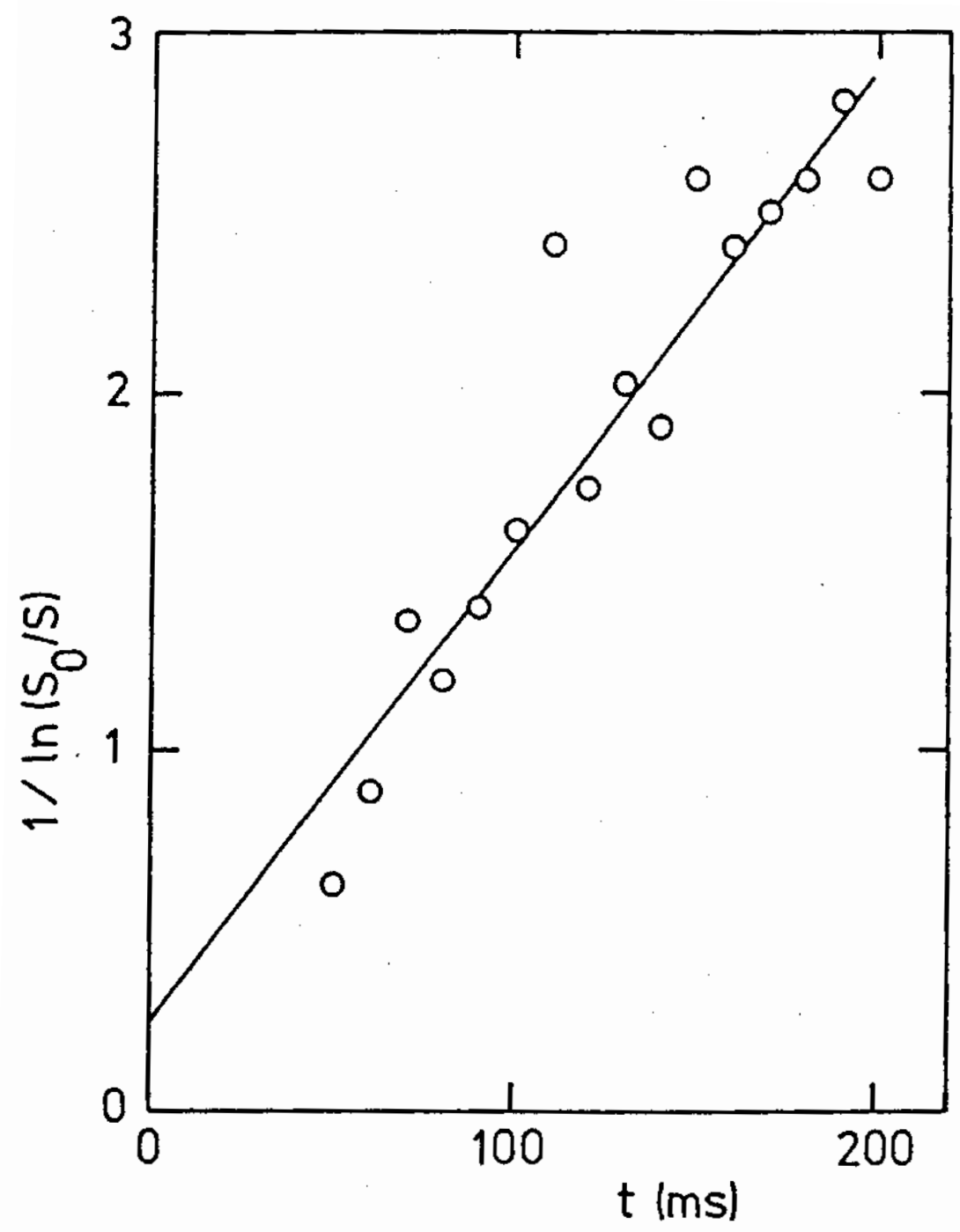

Figure 4: Plot of inverse $\ln \left(S_{0} / S\right)$ against time $t$ over $200 \mathrm{~ms}$ following an expansion through the lambda transition to enable the initial line density at $t=0$ to be extrapolated. 